\title{
Julia Chamard-Bergeron, Philippe Desan et Thomas Pavel (dir.), Les Liens humains dans la littérature (XVIe-XVIIe siècles)
}

Paris, Classiques Garnier, coll. " Colloques, congrès et conférences sur la Renaissance européenne », 2012, 293 p.

\author{
Roxanne Roy \\ Université du Québec à Rimouski
}

Les études réunies par Julia Chamard-Bergeron, Philippe Desan et Thomas Pavel dans cet ouvrage s'inscrivent dans le prolongement d'un colloque tenu le 29 avril 2011 au Centre de l'Université de Chicago à Paris. Les collaborateurs s'interrogent sur la nature, le fondement et la représentation des liens humains dans les textes littéraires français, italiens et espagnols, de la Renaissance à l'âge classique. La richesse de cette problématique ne fait aucun doute puisque les figurations 
littéraires du lien amical, amoureux, filial, marital, social, voire politique invitent à repenser les notions d'intimité, de familiarité, d'opposition entre vie privée et vie publique sous l'Ancien Régime. Il en résulte que la question des liens humains, de leurs transpositions textuelles et des mises en scènes fictionnelles auxquels ils donnent lieu permet «d'éclairer les rapports entre mœurs et belles-lettres » (4e de couverture).

L'ensemble du volume forme un tout d'une remarquable cohérence, chaque article semblant compléter celui qui le précède tout en apportant un éclairage différent sur la problématique commune. En effet, on note une étroite communauté d'esprit entre ces chercheurs européens, américains et canadiens de toutes les générations, ce qui tient sans doute au fait qu'ils puisent aux mêmes sources antiques, philosophiques et morales, revisitant chacun à sa manière les considérations d'Aristote, de Platon, de Cicéron, d'Épicure, de La Boétie et de Montaigne en particulier, tout en prenant appui sur les travaux plus récents de Giorgio Agamben (L'Amitié) et de Jacques Derrida (Politiques de l'amitié). La complicité intellectuelle est encore accentuée par les renvois en notes infrapaginales aux différents travaux menés par plusieurs d'entre eux, ceux de Thomas Pavel, de Phlippe Desan, d'Hélène Merlin-Kajman, d'Éric Méchoulan ou de Larry F. Norman, pour ne donner que quelques exemples. Des préoccupations similaires se retrouvent donc au cœur des réflexions proposées par ces chercheurs, qu'il s'agisse de mettre au jour les rapports étroits entre amitié, utilité et intérêt, de souligner la dimension politique, éthique, morale et esthétique des liens humains, de réfléchir plus globalement sur le concept de vertu humaine, ou alors d'analyser la logique du don et du contre-don qui régit la plupart des liens sociaux. 
La première partie de ce volume aborde les écrits des « Moralistes et mémorialistes ». Dans l'article introductif, Jean Balsamo met bien en évidence la visée apologétique des Essais, nous invitant ainsi à y voir une « épopée paternelle » (p. 19), en étudiant la relation noble qui lie Montaigne à son père et à son patronyme. Philippe Desan s'intéresse aussi aux Essais mais cette fois, ce sont les liens qui se nouent entre La Boétie et Montaigne ainsi que la fonction politique inavouée de l'amitié qui retiennent son attention. Pour sa part, Jean-Charles Darmon propose une riche et stimulante analyse de la philosophie néoépicurienne sur l'amitié en tenant compte de ses déplacements, variations et transpositions dans les écrits de Gassendi, de Saint-Évremond et de Sorbière. Emmanuel Bury approfondit cette réflexion sur la complexité des discours sur l'amitié en soulignant avec finesse le dialogue qu'entretiennent les écrits des moralistes chrétiens Pierre Nicole et La Bruyère avec la tradition philosophique. De manière fort convaincante, Hélène Merlin-Kajman montre que les mémorialistes optent pour un style noble ou familier selon que les liens humains dont ils font état dans leurs écrits relèvent de la sphère privée ou publique. En portant une attention particulière à «la substance langagière du lien social » (p. 105), elle vient pallier une lacune de l'histoire littéraire qui «a méconnu la continuité existant entre les styles sociaux et les styles littéraires, et leur interaction réciproque » (p. 126).

Intitulée "Roman, nouvelle, comédie », la deuxième partie de cet ouvrage collectif traite des liens humains représentés dans les œuvres de fiction. À partir de l'étude du topos du mariage clandestin dans les nouvelles françaises des années 1540 et 1550, Laëtitia Dion montre comment la fiction participe à la réflexion entourant le débat qui oppose le 
principe du consensualisme romain défendu par l'Église au principe du consentement parental soutenu par les élites sociales. De son côté, Anne Duprat se penche sur le motif littéraire de la possession amoureuse comme don de soi dans les nouvelles de Cinzio et de Cervantès. La mise en récit et le traitement narratif de ce lien amoureux, qui se décline aussi sur le mode de la prison d'amour et de la chaîne amoureuse, donne lieu à des jeux d'échanges symboliques entre les personnages reposant sur le principe d'obligation mutuelle. Selon Julia Chamard-Begeron, le conflit entre le lien amical et le lien conjugal servirait de principe dynamique à la « Nouvelle du Curieux impertinent » de Cervantès. Plus précisément, « l'enjeu dramatique de la nouvelle révèle la tension entre le modèle de l'amitié parfaite, conçu par l'Antiquité et relayé par la Renaissance, et le modèle chrétien du mariage » (p. 188). C'est avec un grand intérêt qu'on lit l'article de Françoise Lavocat. Constatant l'omniprésence des catastrophes naturelles dans les romans de la première moitié du XVIIe siècle, elle pose l'hypothèse selon laquelle «les auteurs cherchent à capter le caractère grandiose et l'impact émotionnel de la catastrophe tout en les inféodant à la pensée du roman qui érige l'amour en valeur suprême » (p. 204). S'ensuit une démonstration en trois temps où l'on voit que les catastrophes servent surtout à mettre en relief la conduite exemplaire du héros et son dévouement amoureux, que le lien amoureux prévaut sur le sort de la collectivité affectée par le désastre, que l'amour de l'humanité ne peut l'emporter que lorsqu'on invente un nouveau type de personnage, soit le témoin fictif non amoureux se retrouvant au cœur d'une catastrophe naturelle. Guiomar Hautcoeur cherche à comprendre les liens du sang, qui reposent sur une alliance entre liens lignagiers et humains, en analysant le motif de la 
reconnaissance par leurs parents des enfants perdus à la naissance. Pour ce faire, il se penche sur trois romans clés qu'il résume et commente : Les Éthiopiques, L'Astrée, puis Pierre et Jean. C'est sous le prisme de la notion d'intimité que Larry F. Norman étudie les liens sociaux qui se nouent dans les pièces de Molière, en privilégiant trois scènes tirées du Misanthrope. La problématique choisie permet « d'examiner quelques enjeux essentiels de l'expression verbale et théâtrale » (p. 246), en soulevant la question du style intime de même que celle de la dramaturgie des conflits intimes qui structurent la pièce. L'auteur élargit encore ses réflexions en englobant le lien qui attache l'auteur à son public, annonçant du même coup la thématique abordée dans la dernière partie du volume.

En guise de conclusion, la troisième partie envisage les «Liens littéraires» qui se tissent entre auteurs, lecteurs et personnages. Éric Méchoulan nous invite à concevoir l'adresse à l'ami lecteur comme une stratégie employée par l'auteur pour bien prédisposer le lecteur à l'égard de son livre et en orienter favorablement la réception. Dès lors, le rapport que le lecteur entretient avec l'auteur repose sur la réciprocité et la solidarité affective, selon la logique du don et du contre-don : «l'auteur donne son livre, le lecteur reçoit avec plaisir et redonne à l'auteur en reconnaissance sociale » (p. 266). Le volume se clôt sur les réflexions de Thomas Pavel, qui cherche à comprendre comment un lecteur peut être transporté par des vers et éprouver de l'admiration pour un personnage en dépit de la distance historique qui les sépare. Invoquant tour à tour les propos de Giambattista Vico, d'Adam Smith, d'Edmund Burke et de Schleiermacher, il convient finalement avec Johann Gustav Droysen que «la sympathie morale, à la fois intuitive et 
intellectuelle, opère à travers les clivages historiques et culturels » (p. 285).

$\mathrm{Au}$ terme de cette lecture, on peut affirmer qu'il s'agit là d'un ouvrage incontournable pour quiconque s'intéresse de près à la question des liens humains dans la littérature de l'Ancien Régime. On se saurait trop insister sur la qualité intrinsèque des articles, la rigueur de leur raisonnement et leur élégance stylistique. 